\title{
Kotaview: Simulating Traditional Korean Architecture Interactively and Intelligently on the Web
}

\author{
Jie Eun Hwang ${ }^{(*)}$ and Jin Won Choi ${ }^{(\#)}$ \\ Yonsei University, Department of Housing \& Interior Design, Seoul, Korea \\ E-mail: ${ }^{(*)}$ curiozen@yonsei.ac.kr \\ ${ }^{(*)}$ jchoi@ yonsei.ac.kr
}

\begin{abstract}
Traditional architecture is an important topic, not only for finding local identities from traditional heritages but also for representing the traditional identities. It is especially true because locality issues are critical in this global era. Nevertheless, the structure of traditional Korean architecture has not been fully explored in a systematical or computational manner. Furthermore, this information is not managed efficiently even though the technology for managing information effectively is quite widely available and the information could be used in many application areas. Currently all but a few experts keep their knowledge exclusively and transfer it to few people in a limited and traditional manner. This study thus selects the Internet as an educational environment for practical reasons and explores a way of structuring construction knowledge and building information of traditional Korean architecture. That will be the first step to manage information of traditional architecture. In order to simulate traditional buildings in advanced and intelligent ways, we apply a computer technique, called data modeling. We develop a robust data model to effectively represent the building. For data modeling, we select a well-known building, Buseoksa Muryangsujun as a
\end{abstract}

prototype, which is one of the oldest wooden structures in Korea. We first build an accurate three-dimensional model of the building. And then analyze its building components and their connectivity by focusing on the capital order system, called Gongpo. We also suggest a user interface model operated through the data model. The interface provides several components and functionality such as viewing each architectural component, simulating the components' connectivity interactively, and retrieving architectural information. The study shows a diagram of the wooden construction data model and some rules to explain components' connectivity, and a user interface model. This is currently being integrated into the KotaView system based on two environments: a web-based solid modeler(called WebMod) and a web-based VR engine (Active World ${ }^{\mathrm{TM}}$, $\mathrm{EON}^{\mathrm{TM}}$ ).

KEYWORDS: data model, traditional Korean architecture, tutoring system, Buseoksa Muryangsujun

\section{INTRODUCTION}

New trends for this millennium include both globalization and localization at the same time. The new paradigm of localization requires local and national identities that have frequently been 
ichim 01

CULTURAL HERITAGE and TECHNOLOGIES in the THIRO MTLLENNIUM

ignored during the modern industrial era of the last decades. Finding national identities from its traditional heritages might be an important research issue. In Korea, having 5000-year-history, both historic preservation and inheritance are deserved to important issues.

\section{Korean Context}

Most Koreans believe that they did not have much time to explore and deeply understand their ancestors' works during the last decades. In fact, on traditional Korean architecture it is noticed that its information has not been fully explored in a systematical or computational manner, and also is not shared efficiently. Information sharing might be, we believe, an essential part or at least the first step to understand traditional architecture. Currently all but a few experts keep their knowledge exclusively and transfer it to few people in a limited and traditional manner.

One of the reasons is due to the inheriting way of traditional architecture relying on the traditional craftman's man-to-man way, rather than a more systematic manner. So it is not so easy to approach the information of the traditional Korean architecture even for architecture students and building experts. Another reason is related to the problem of media with which the information is incorporated. Traditional Korean architecture is based on the wooden construction complicatedly assembled by prefabricated components (Figure 1). But currently the media of most architectural information consist of two-dimensional drawings, inadequate to present prefabricated three-dimensional components. Two-dimensional drawings also have obvious limitations in describing the joints and relations among the components, so it is almost impossible to understand at a glance the whole construction process only referring to them (Figure 2).

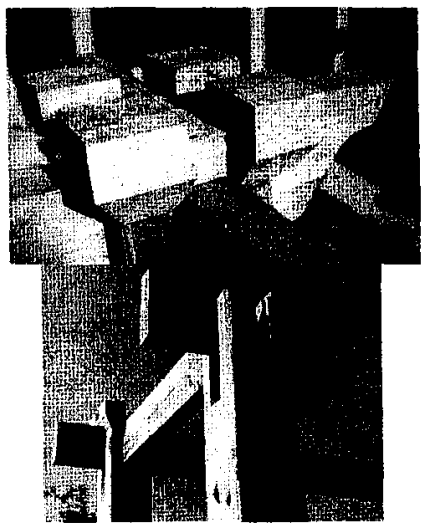

\section{Figure 1: The construction process by assembling prefabricated components}

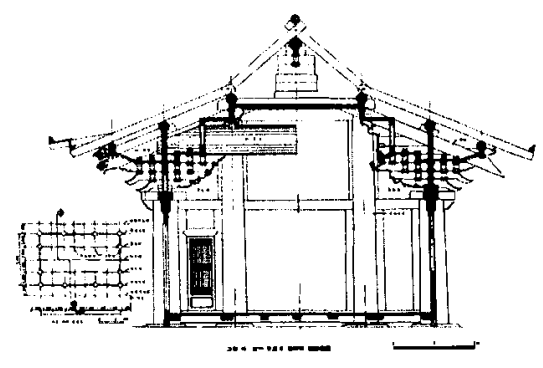

Figure 2: The current media of architectural information

An Innovate Way To Learn Traditional Architecture

The ultimate purpose of the study is to classify the information of traditional Korean architecture in a systematic and computational manner in order to share the information efficiently. Even though the most popular approach to present visual information on the Internet is to use still images and animation clips, we take a different, yet innovative approach. In order to simulate traditional buildings 
in advanced and intelligent ways, we apply a computational technique, called data modeling. A data model means a conceptual model that includes a mechanism to fully describe a certain domain of data in a semantically fluent way.

Also we investigate efficient media forms to present the information of traditional buildings. We already described the problem of the current media for architectural information-two-dimensional drawings. Such a media as fully delivers three-dimensional data could solve the problem mentioned above. If the information of traditional Korean architecture is presented in and shared with three-dimensional data, it is very helpful to understand the building information and to communicate the architectural knowledge with others. Moreover, a design tool can be developed to generate new types of buildings. Finally, on this study, we aim to supply the core technology of managing information of traditional Korean architecture with a prototype system with an effective user interface and a robust data model.

\section{KotaView: a Tutoring System within KotaSys}

The demands for sharing information of traditional Korean architecture occur from various application areas. Grouping the possible application areas, it is classified into approximately four areas: publicity, education, design, and research. In this study, we concentrate on the education field- a tutoring system for practical demands. KotaView is a tentative name of the web-based tutoring system within KotaSys, an integrated information management system of traditional Korean architecture, which we are currently developing. KotaView will be the first effort of the KotaSys development, because the data model included in KotaView is the core module to operate KotaSys, After the data model in KotaView is completed we will extend to develop the other systems in KotaSys, including all of different applications: a publicity system for tourism and publicity, a tutoring system for education, a generative system for design, and a survey system for research.

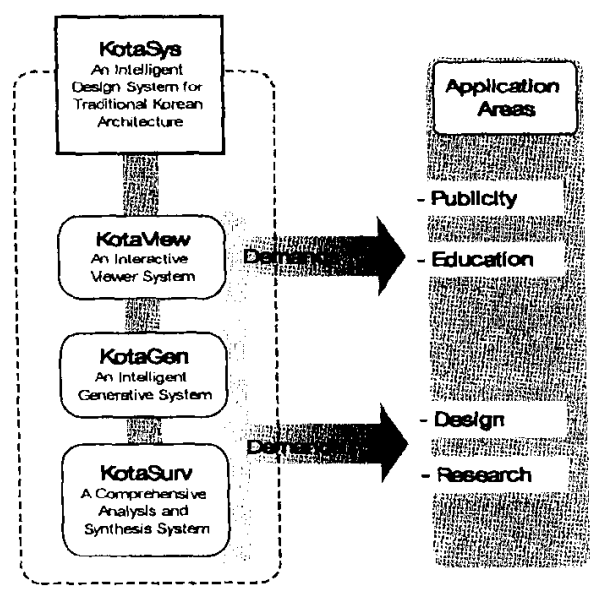

Figure 3: The overview on KotaSys

\section{The Substance of the Study}

For data modeling we select a well-known temple building, Buseoksa Muryangsujun as a prototype (Figure 4). Buseoksa Muryangsujun is known for the second oldest building in the existing Korean traditional wooden structures, and over the past 600 years has never been renovated. So it is proper to regard Buseoksa Muryangsujun as a representative example and prototype of traditional Korean architecture. 
ichim 01

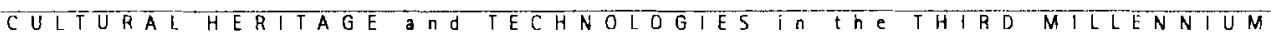

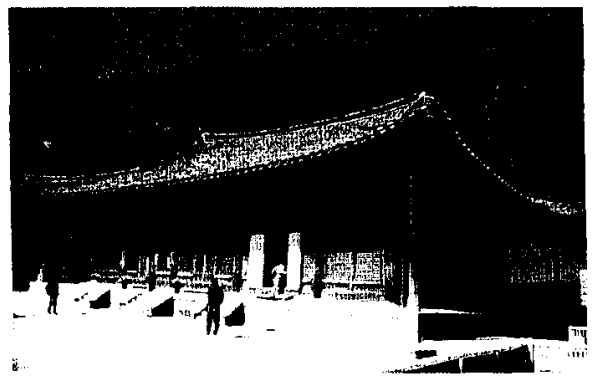

Figure 4: The exterior view of Buseoksa Muryangsujun

We first build an accurate three-dimensional model of the building with an aid of a traditional building expert, categorize its building components, and then analyze their forms and design determinants by focusing specifically on the capital order system. Like the Greek order system, the Korean order system, called Gongpo (Figure 5), is both structural and ornamental, and plays a key role in constructing the wooden structure.

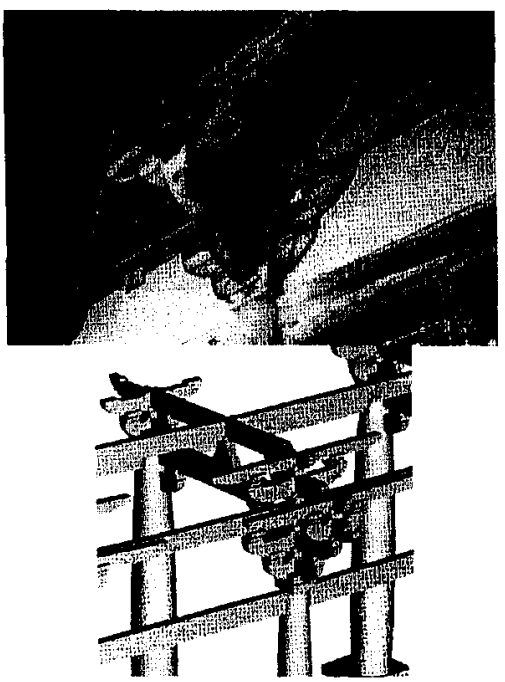

Figure 5: The Gonpo structure, a capital order system

\section{A USER INTERFACE MODEL OF KOTAVIEW}

A Synopsis of the KotaView, Tutoring System

KotaView is a web-based tutoring system of traditional Korean architecture we are currently developing. Even though navigation is a main feature in the system to experience the structure of traditional Korean architecture with 3D objects similar to the real world, as a tutoring system, it requires effective instruction programs, administration techniques, and cumulative guidance. The synopsis for operating KotaView is described in Table 1.

The typical interface of the Kotaiew is composed of five main parts: 1) a main menu frame to contain the subjects of the whole system, such as preferences, lectures, courses, boards, etc.; 2) a sub-menu bar to contain complete categories to classify the main menu items, whenever each main menu item is selected and refreshed automatically; 3) an embedded viewer to show the real-time simulation of the construction, walk-though animations, graphs, whatever the selected item to show visually; 4) a movie player to show interactive movies including some necessary information; and 5) a prompt window to show real-time messages

\section{Tutoring Components}

A Tutoring Component is one of the important operations. When a user selects an item of the Tutoring Component in the main menu, a submenu bar is loaded to contain the categories of the components, such as foundation, column, order, roof, and finish. Each category has sub-categories or groups of components. By clicking the sub-menu items, the user can roughly understand the assortment of building components. When one chooses a 
ichim 01

CUITURAL HE RITAGE âd TECHNOLOGTES Tn the THIRD MILLENNIUM

component in the sub-menu bar, the window is updated with the new contents of the selected item. The viewer presents a 3D form of the selected component. The user can simulate it similarly to the real world. Users can zoom, pan, or rotate components selected. When the mouse point is located in the critical points such as a connection point, a special cutting point, etc., the prompt window shows the information and more hyper links related to the situation. The movie player shows movies to contain the information of the selected component; e.g. the assemblage orders in selected group of components, the process to carve the component, and the method to joint. In case of a movie to show the connection by assemblage order, users can change the position of a viewpoint wherever they want to see, and move to other sessions through the hyperlinks in the movie.

Table 1: The synopsis of KotaView

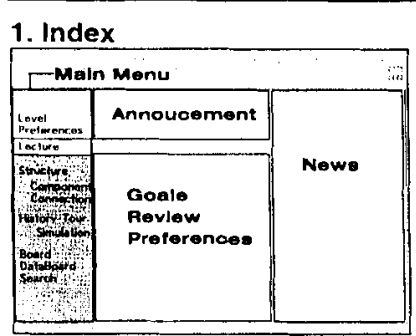

When users login the system, first they meet an index page that can be personalized later. It consists of a main menu to contain the subjects of the whole system, and personalized information; announcements, goals and reviews of learning, system preferences, and news.

\section{Tutoring Components}

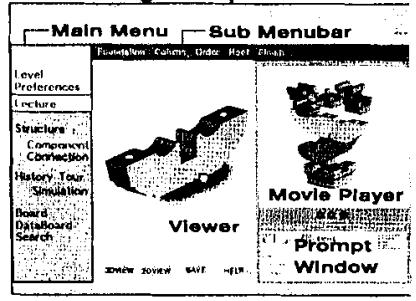

A Tutoring Components page presents all the components composed of typical Korean traditional architecture. A sub-menu bar to contain complete categories to classify components, an embedded viewer to show the real-time simulation of a selected component.

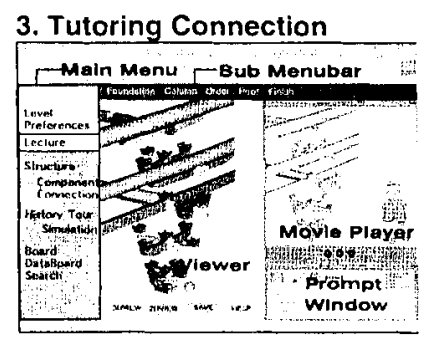

A Tutoring Connection page presents all the connection classified in several connecting groups. Users get experience on connecting and assembling components in a connecting group selected. The typical interface is very similar to the Touring Component page. 
ichim 01

CUITURAT HERTTAGE and TECHNOLOGIEST T TC THTRO MILLENNTUM

\section{Touring Heritage}

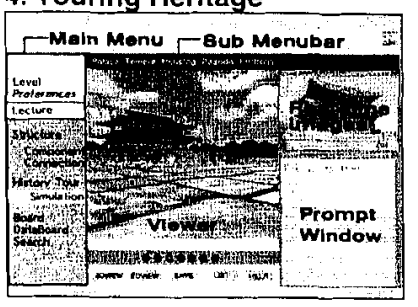

A Touring Heritage page consists of an interactive walk-though simulation. There are several categories of classitying Korean architectural heritages on the sub-menu. When a user selects one of them, the information of a selected building is loaded.

\section{Instructor's Control Mode}

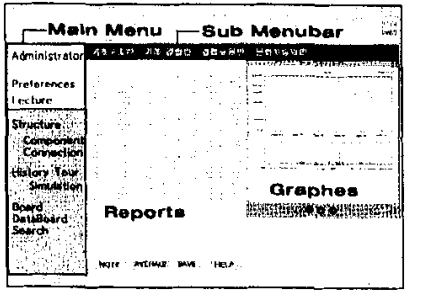

After a user login the system as an instructor, s/he can control and analyze events generated by students. The information of students (e.g. learning levels. performances, study results, frequencies, etc.) is updated in the user database

\section{Student's Control Mode}

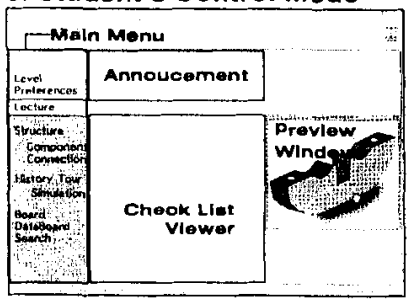

When a student logs out, a Student's Control Mode page is automatically loaded. The student user can check and review the tolal contents of learning one by one. The items students have ever learned will be update in the user database.

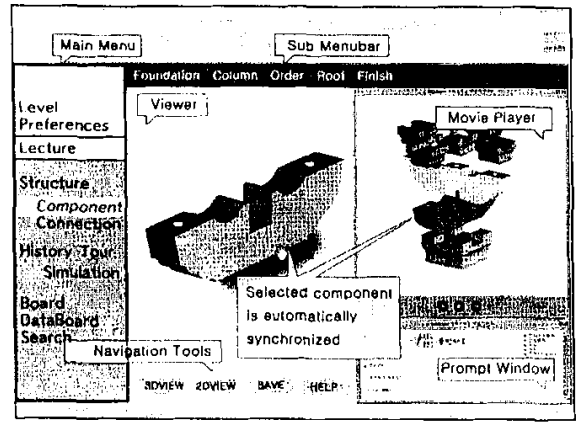

Figure 6: The schematic interface of Tutoring Components

\section{Tutoring Connection}

A Tutoring Connection is also the key operation. A schematic interface is quite similar to a page of Tutoring Components. The viewer would have more functions to show the connecting way efficiently. For example, a user can move a component and snap it to other component in the selected connection group. Because KotaView contain the data model, some distinctive operations are possible as follows: to decide user-making structure's accuracy, to find out disjointing order, and to assemble the selected components under some constraints fixing the position of a 
component. Furthermore, the user could make a new form by the generative rules. This is one of the basic concepts in the generative methods included in a generative version of KotaView.

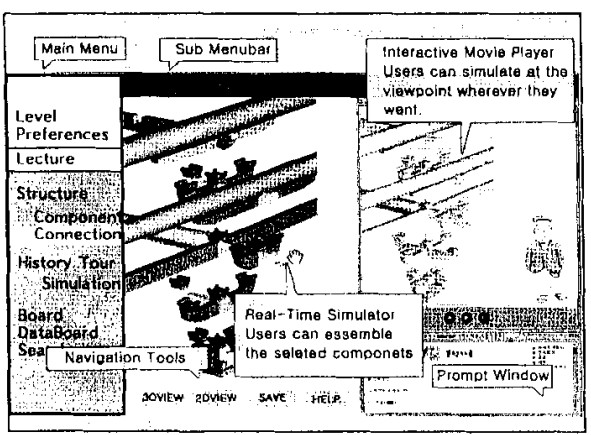

Figure 7: The schematic interface of Tutoring Connection

The core operation of KotaView would be Tutoring Components and Tutoring Connection. It is because those operations are closely related to the data model. That is also why the operations are distinguished for tutoring and sharing the information of traditional architecture. So a robust data model is an indispensable premise, which contains the architectural knowledge, building components, the connectivity between components, and their connectivity rules.

\section{THE DATA MODELING OF GONGPO FOR KOTAVIEW}

The Analysis of the Gongpo Structure The component shapes to compose a Gongpo do not always correspond to the names and types. Some components have different names or roles in spite of their same identical shape, while some have a same role with different shapes. Thus, for the study, it is more efficient to regulate shape-types, component types per each component. We also specify simplified terms instead of complicated real names. Table 1 shows the list of component

types.

Table 2: The list of component shapes

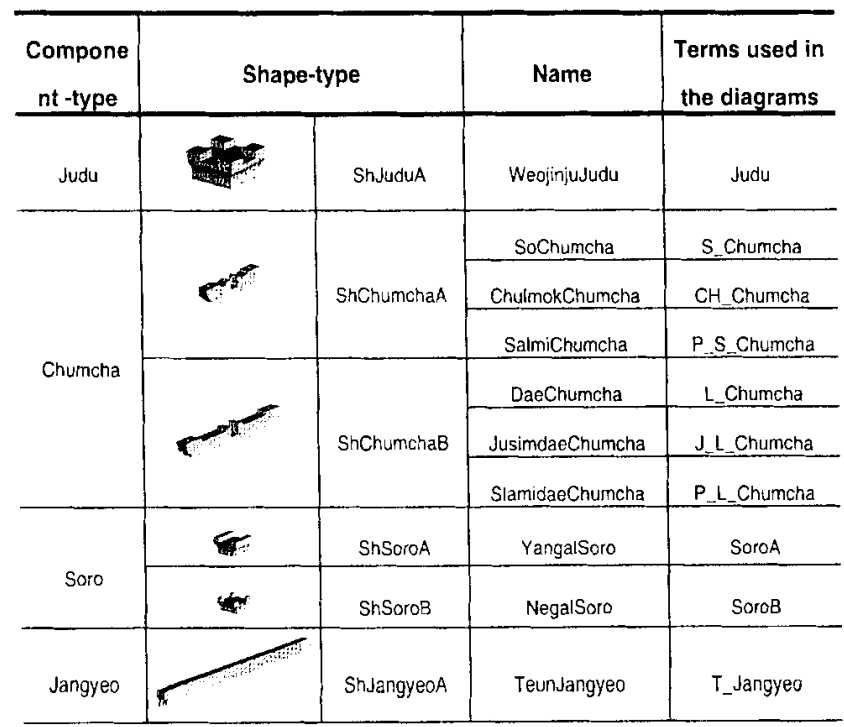




\section{ichim 01}

CULTURAT HEATTAGE AO J TECHNOLOGTES IT ThE THTRO MILLENNTUM

\begin{tabular}{|c|c|c|c|c|}
\hline & & ShJangyeoB & DanJangyeo & D_Jangyeo \\
\hline Bo & & $S h B \circ A$ & TeuBo & T_Bo \\
\hline \multirow{2}{*}{ Chobang } & & ShChobangA & \multirow{2}{*}{ Chobang } & \multirow{2}{*}{ Chobang } \\
\hline & & ShChobangB & & \\
\hline \multirow{2}{*}{ Dori } & & \multirow{2}{*}{ ShDoriA } & JusimDori & J_Dori \\
\hline & & & ChulmakDori & $\mathrm{CH}_{\text {_Dori }}$ \\
\hline
\end{tabular}

After listing up the component shapes, we unfold all of the components in a Gongpo and analyze them by the construction orders. There could be some repetitive rules in the assemblage orders such as in Jegong. In general, a Gongpo is composed of a Judu, Jegongs, Chobangs, DanJangyeos, and JusimDories. A Jegong is a sub-structure composed of repetitively layered subsets such as ChoJegong, YiJegong, SamJegong, and SaJegong. Each Jegong is also made up by a repeated rule of assembling. Now we develop several schema diagrams representing all the components and sub-structures with the connecting rules.

On the basis of the shape regulation and connection rule, we can develop the structure of Gongpo visually as shown in Figure 8 . The gray circle represents a component and the white circle represents a sub-structure or a group of components. The lines represent the composition hierarchy and connectivity. The diagram is a basic framework to analyze Gongpo in this study. 
ichim 01

CULTURAL HERTIAGE a Td TECHNOLOGTES TR ThE THTAO MILEENNTUM

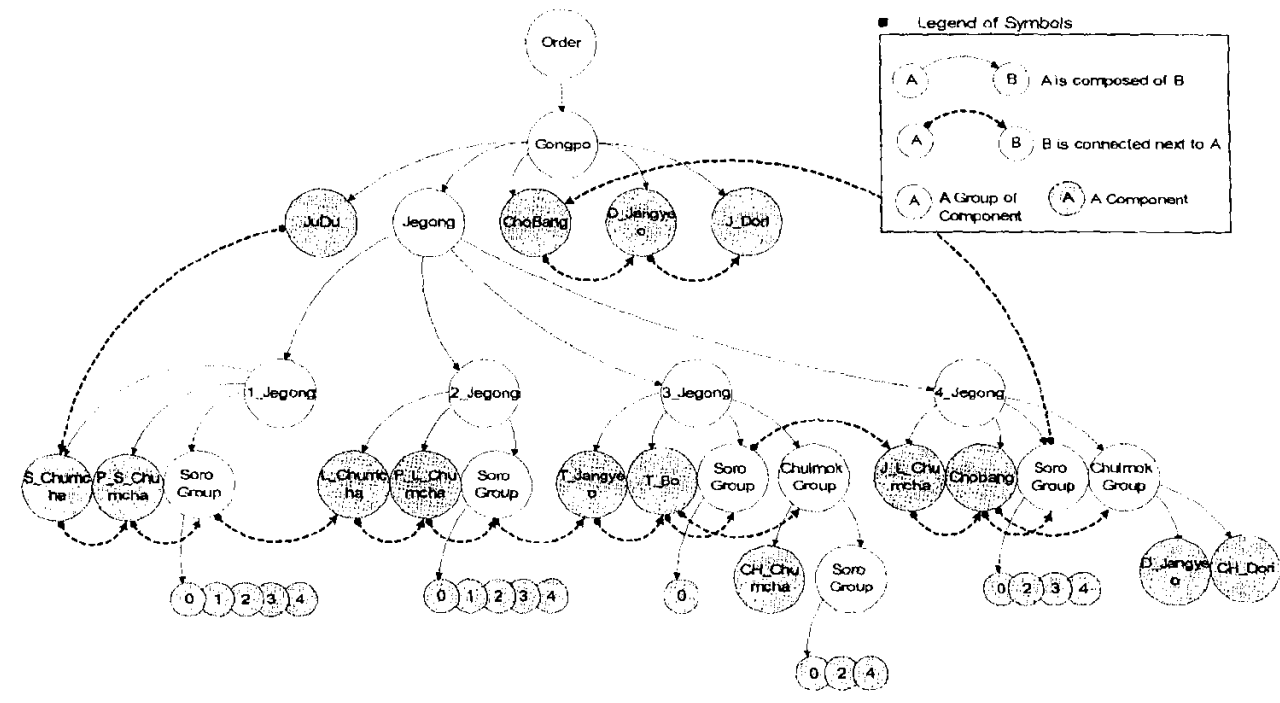

Figure 8: The basic frame diagram for analysis

Types of Component Shapes

Figure 9 is a diagram featuring the types of component shapes, which we already arranged in Table 1. There are total 11 shape types in Gongpo, and one component must match one shape type. The type of shape is sorted only by the shape uniqueness not to concern with name, role, and position. 


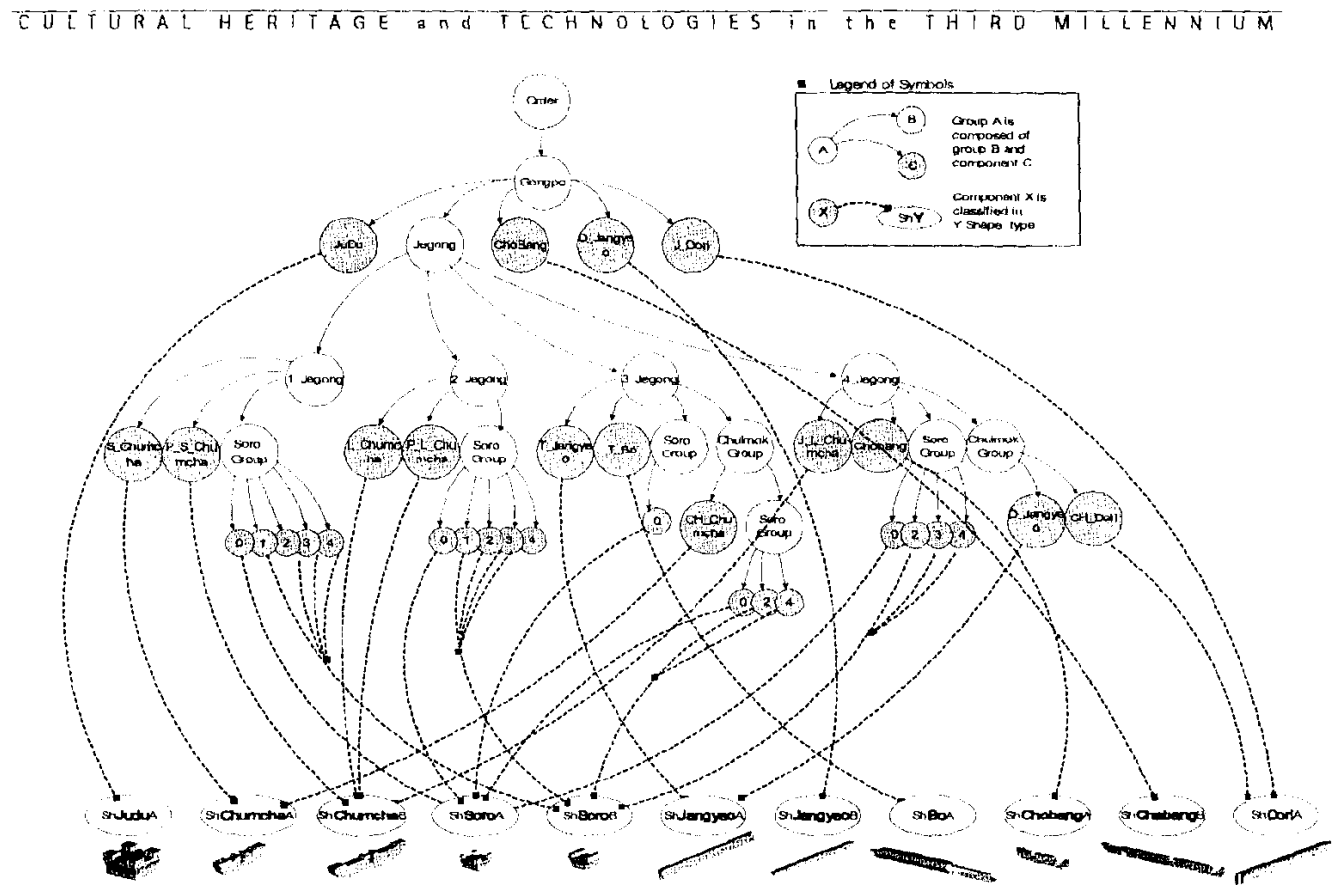

Figure 9: The schematic diagram for types of components

Types of Connection Roles by the Assemblage Orders

While connecting components with the assemblage order, we can recognize some components (or groups) determine the shape of the whole building, and the others attach two more determined components. We call that a constructor and a connector. The concepts of connectors and constructors would play a key role in generative rules for a later version of a KotaGen, a generative system. In Figure 8, the gray circle represents a connector, and the whitc circle represents a constructor.

In Jegongs we can particularly figure out the repetitive nulcs of assembage. A subset of Jegongs is composed of both Dori-directional components and Bo-directional components, a Soro group evenly, and a Chulmok group occasionally. Figure 8 presents this rule. 


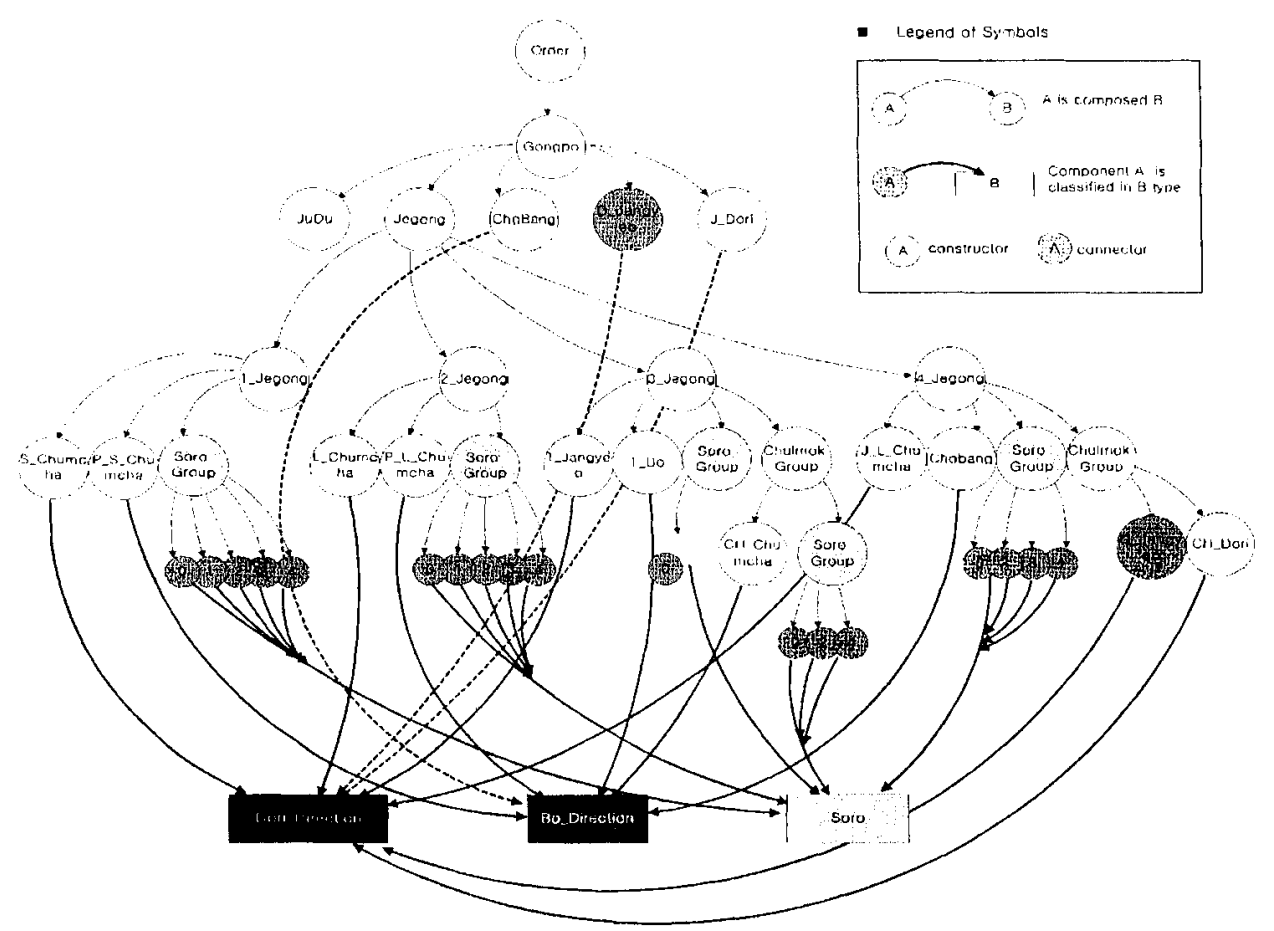

Figure 10: The schematic diagram for types of connection roles by the assemblage orders

The Classification of Components

After considering all the analysed factors, we can classify the components of Gongpo (Figure 13). We develop possible component classes and investigate their dependencies on each other, and then link them to each other based on the rules already described above. A component or a group of components could define a class, and we symbolize it as unit box (Figure 11). A class contains various members describing properties of the class, relationships among the other classes.

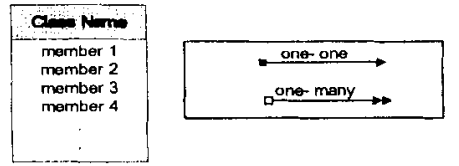

Figure 11: Symbols for diagram of classification

The relationship between classes is concern with connectivity between components. As Figure 11 shown, there are two types of relationships. One is one-to-one relationship and the other one-to-many relationship. One-to one relationship means a class has an instance linked with another class. And one-to-many relationship means a class 
TULTURAL HERTTAGE AT TECHNOLOGTES In TAE THIRT MILLENNIUM

has many instances linked with another class. For example, in case of Figure 12, Jegong' class, KaJegong includes 6 members; type, name, position, is_constructor, fisrt_soro, prejegongpart. Of all these members, type, name, position, and is constructor indicate the properties of KaJegong. And first_soro, and prejegongpart make matches the other classes, KaSoro and KaJegongPart. A Jegong has many Soros, so the relationship between KaJegong and KaSoro is one-to-many relationship.

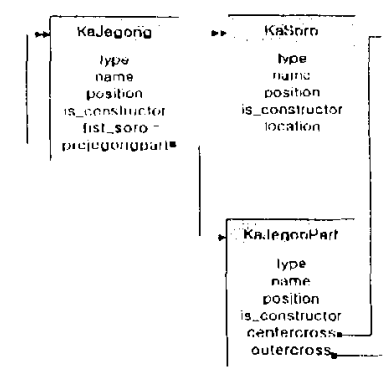

Figure 12: The relationship of KaJegong class
The classification will be implemented to develop the KotaSys system, which manages information of traditional architecture.

The Relationships between
Components component classes, we develop more multi-meaningful diagram including complex relationships of connections. The possible representations are as follow: 1) the types of components; a category, a prototype, a group of components, and a component; 2) the types of components roles; constructor or connector; 3) the types of quantities; singular type or plural type; 4) the conditions of connection; 5) the adjacency and connectivity between components; and 6) the assemblage process. Figure 14 shows the complex relationships between components (or groups) by the various notations of the paths and explains types and statues of classes. Figure 15 illustrates the whole feature of of the Gongpo structure corresponding to component classes visually. 
ichim 01

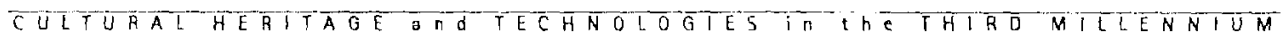

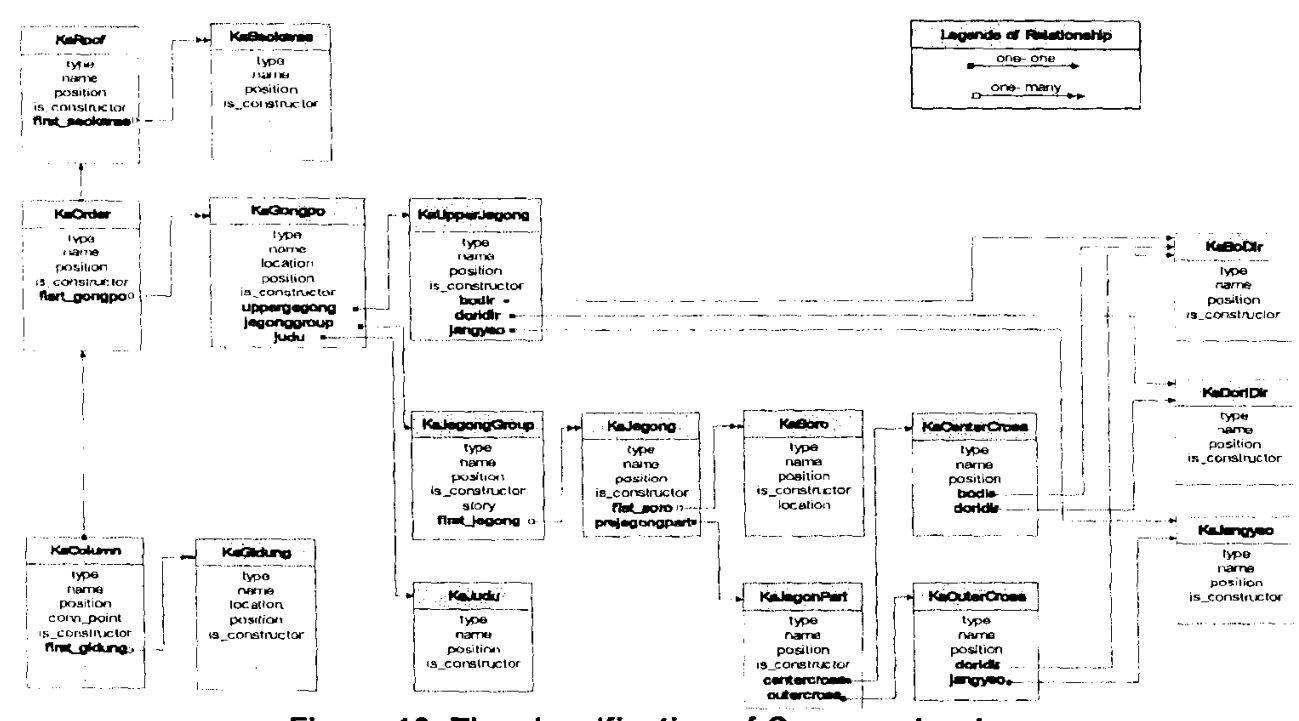

Figure 13: The classification of Gongpo structure 


\section{ichim 01}

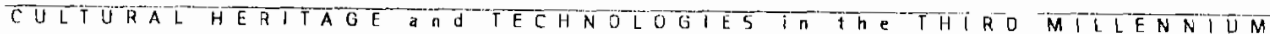

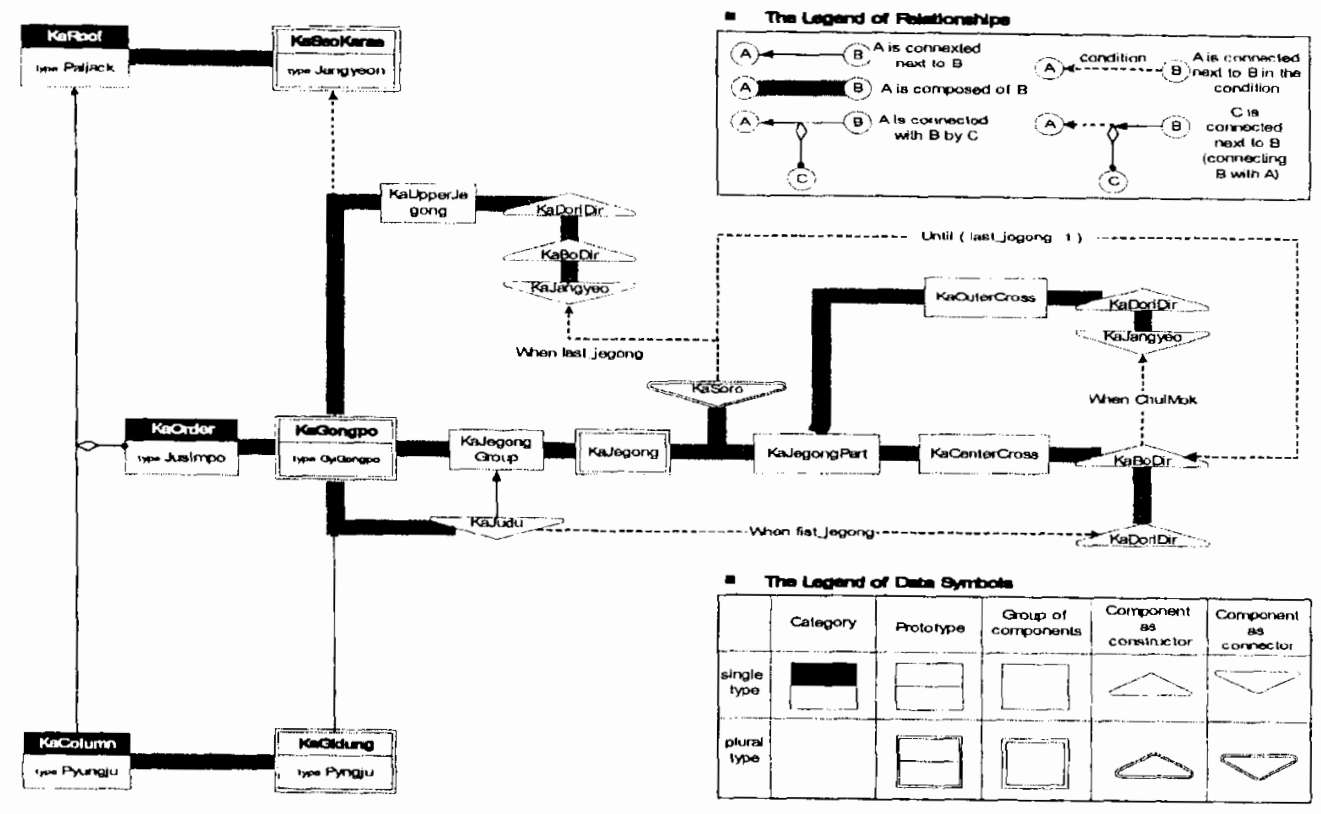

Figure 14: The relationship between component classes 


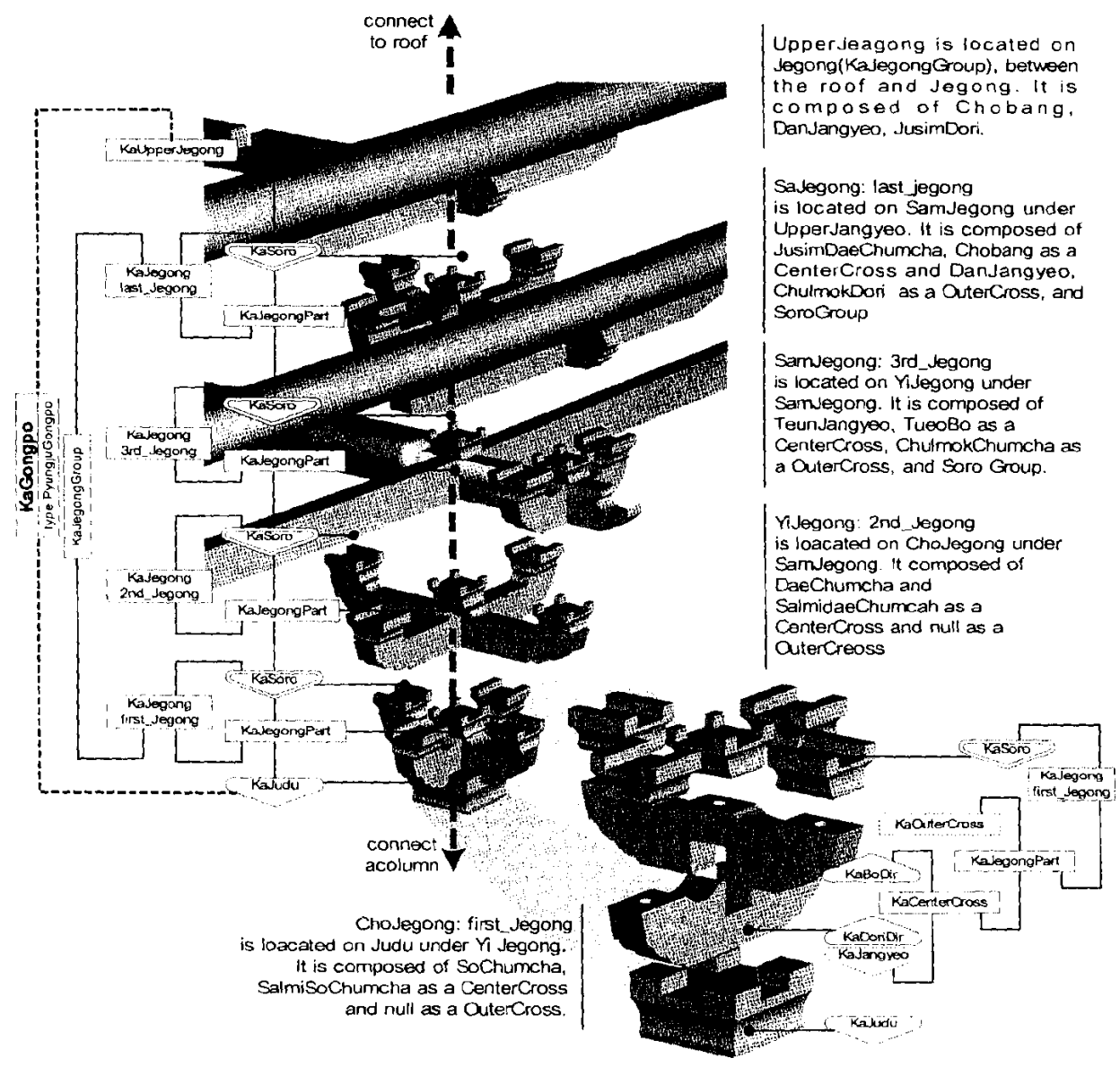

Figure 15: The whole feature of the Gongpo structure corresponding to component classes

\section{STRATEGY OF IMPLEMENTATION}

We are making progress to implementation KotView with two approaches. One is the academic approach and the other is more practical approach.

We have currently developed a web-based solid modeler, called WebMod, which will be a main geometric engine of KotaView (Figure 16). We are currently implementing the whole structure of Gongpo class in WebMod. There are, however, a few limits to complete the KotaView as a tutoring system. We supplement the limits in WebMod by the commercial VR engincs such as Active World ${ }^{\mathrm{TM}}$ (Figure 17) and EONTM(Figure 18). Each engine has different features, merits and 
demerits. Once the data model is confirmed in WebMod, it would be transferred. We then implement the whole feature of KotaView.

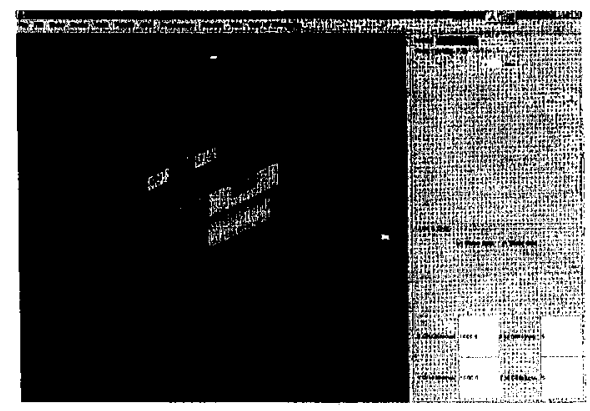

Figure 16: WebMod: the main geometric engine

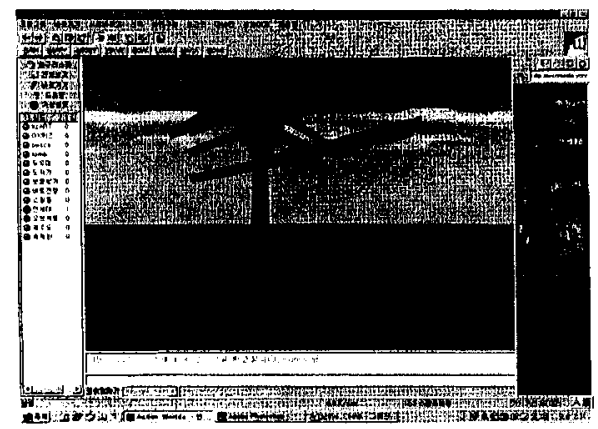

Figure 17: The development in Active World ${ }^{\mathrm{M}}$

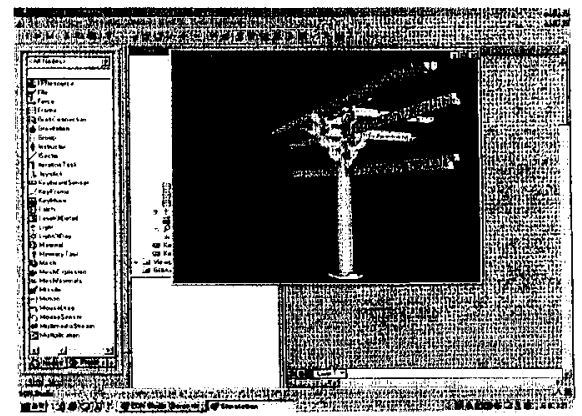

Figure 18: The development in EONTM

\section{CONCLUSION}

This study is to investigate ways of sharing information about traditional Korean architecture for historic preservation and inheritance. Of all demands for the information of traditional architecture, we concentrate on educational field, because it would be the core framework to be able to extend the other applications. We develop a robust data model in order to effectively and intelligently describe traditional Korean architecture. To do this, we analyzed and classified the components of the Gongpo structure in Buseoksa Muryangsujun as a prototype.

- In the Gongpo structure, there are 11 types of component shapes, and each component shape is closely related to the connecting location and its role.

- Each component in the Gongpo structure has the properties caused by the assemblage rule. Especially in Jegong, we find out a repetitive assemblage rule among the composition of Bo-directional component, Dori_directional component, and Soro group. The connection role in assemblage process characterizes the component, if it is a constructor or a connector. This rule is a start point to build the data model.

- The Gongpo structure is successfully classified into a component or a group of components, and the relationship between the classes can be well described.

- Gongpo structure has assemblage logics and in turn each component has connection logics to define shapes of the Gongpo structure. These logics are the basis of new-generative logics, and can be extend to develop the generative 
system creating new Gongpo structures.

- The web-based tutoring system, KotaView, is intelligent enough to understand the structure, to present it visually, and to control the user 's learning point.

\section{FURTHER STUDY}

Further research issues identified are as follows: 1) expanding the data model to explain the whole building's structure; 2) developing completely a VR-based tutoring system to simulate the construction of traditional Korean architecture; 3) developing a generative design system, which can intelligently generate a new type of wooden buildings that could actually be built; 4) developing a survey system, which is tightly combined with the database of traditional Korean architecture, and finally; 5) completely developing KotaSys, an intelligent information managing system of traditional Korean architecture.

\section{ACKNOWLEDGMENTS}

The 3D objects included in this paper were drawn by Mi-Jung Kim, a Ph.D. candidate at Yonsei University and Jie-Yong Shin in Hanok Cultural Center

This paper included a part of the research results from the VRRC research project funded by Korea Science and Engineering Foundation.

\section{REFERENCES}

1. Chang, G.I.: 1987, Wooden Structure, Bosunggak, Seoul.

2. Chang, G.I.: 1986, Korean Traditional Architectural Glossary, Bosunggak, Seoul.

3. Eastman, C.E.: 1994, A Data Model for Design Knowledge, Automation in Construction, 3, pp.135-147.

4. Kim, U., Choi, J.W., and Kim, S.A.: 1999, A House Design Automation System Based on the "Design-by Novice", Architectural Research, Journal of the Architectural Institute of Korea, 1(1), 23-30.

5. Mitchell, W.J.: 1994, Artifact Grammar and Architectural Invention, in Tzonis A. and White I. (eds), Automation Based Creative Design,, pp.139-159

6. Shin, y.h.: 2000, Our Housing Building (Woori Hanok), Hyunamsa, Seoul

7. Stiny, G.: 1994, The Pedagogical Grammar, Automation, in Tzonis A. and White 1, (eds), Automation Based Creative Design, pp.129-138.

8. Active World ${ }^{\mathrm{TM}}$, http://www.activeworlds.com,

9. EONTM, http:// http://www.eonreality.com

\section{ABOUT THE AUTHORS}

Jie Eun Hwang is enrolled in the master program in the Department of Housing \& Interior Deign at Yonsei University, Seoul, Korea. She is a member of the CAAD research lab., Archmedia(http://archmedia.yonsei.ac.k $r$ ). Currently research involved in includes as follows: applications of virtual reality in architecture such as the development of KotaSys; and the development of three-dimensional modeler based on GIS.

E-mail: curiozen@yonsei.ac.kr

Jin Won Choi is an assistant professor in the Department of Housing \& Interior Deign at Yonsei University, Seoul, Korea. He was granted a doctorate in 
ichim 01

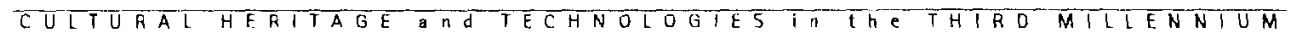

architecture at the Ohio State University, in 1995. He is the director of the CAAD research lab., Archmedia (http://archmedia.yonsei.ac.kr). Current research interests include architectural computing, design collaboration, intelligent CAAD systems, building simulations, computer graphics, virtual reality in architecture, and solid modeling.

E-mail: jchoi@yonsei.ac.kr 